\title{
Medical Image Of The Week: Metastatic Testicular Cancer
}

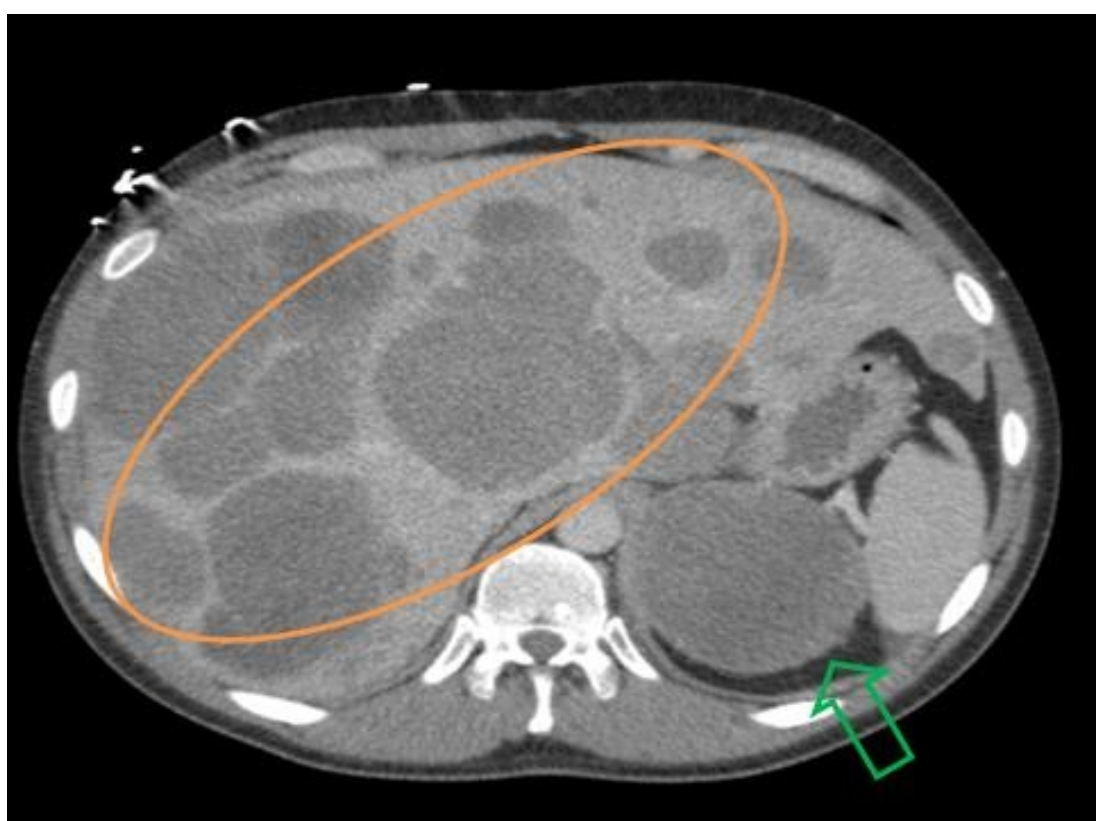

Figure 1. Axial image of the abdomen demonstrating multiple hypodense hepatic lesions (orange oval) and a large left adrenal mass (green arrow).

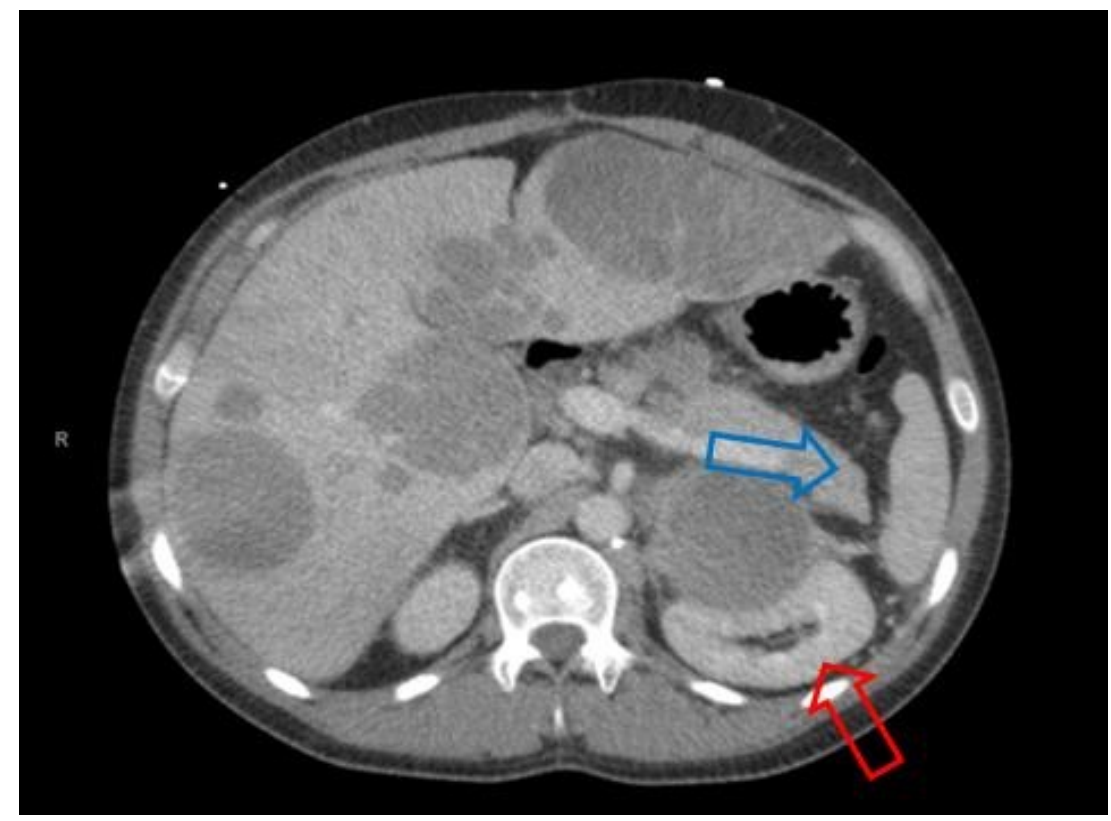

Figure 2. Axial image of the abdomen with innumerable hepatic metastatic lesions and a mass arising from the left adrenal gland resulting in compression of the left kidney (red arrow) and lateral displacement of the spleen (blue arrow). 
A 30 year-old man with metastatic embryonal testicular cancer was admitted to the hospital with severe abdominal pain. A contrast enhanced CT of the abdomen demonstrated large metastatic burden throughout the liver and the left adrenal gland (Figures 1 and 2). The mass arising from the left adrenal gland caused significant mass effect. The left kidney was compressed, though without hydronephrosis, and the spleen was displaced laterally. Renal and hepatic functions were preserved. His pain was controlled with opioids and oral steroids with significant improvement.

Michael Debo DO${ }^{1}$, Linda Snyder $\mathrm{MD}^{2}$, S. Michelle Rhodes $\mathrm{MD}^{3}$.

${ }^{1}$ Department of Internal Medicine, Genesys Regional Medical Center; Grand Blanc, MI ${ }^{2}$ Departments of Medicine, Pulmonary and Critical Care and Palliative Medicine, University of Arizona Medical Center; Tucson, AZ

${ }^{3}$ Departments of Emergency Medicine, Geriatrics, Palliative and General Medicine, University of Arizona Medical Center; Tucson, AZ 